Gynäkologe 2017 · 50:242-243

DOI 10.1007/s00129-017-4043-1

Online publiziert: 2. März 2017

C) Springer Medizin Verlag GmbH 2017

CrossMark
Nikolaus de Gregorio - Wolfgang Janni

Universitätsfrauenklinik Ulm, Ulm, Deutschland

\title{
Das Vulvakarzinom, (leider) eine Erkrankung der Zukunft?
}

Der Schwerpunkt der Ihnen vorliegenden Auflage ist dem Thema Vulvakarzinom gewidmet.

Dabei handelt es sich um eine Erkrankung, mit der wir als GynäkologInnen sowohl im ambulanten als auch im stationären Bereich in Zukunft häufiger konfrontiert werden und die das Zervixkarzinom vermutlich bald in der Inzidenz übertreffen wird. Über die Gründe dafür wird Ihnen der Artikel von Ahmet El Balat et al. Auskunft geben.

\section{) Nach wie vor steigt die Inzidenz des Vulvakarzinoms, vor allem bei Jüngeren}

Trotzdem gehört das Vulvakarzinom zu den seltenen Erkrankungen, die Durchführung von prospektiven, randomisierten Studien ist schwierig bzw. kaum möglich, die Datenlage häufig dünn. Mit der Publikation der AGO-CARE-1-Studie durch Sven Mahner et al. [1] konnte mit über 1200 Patientinnen die größte retrospektive Untersuchung $\mathrm{zu}$ dieser Entität weltweit aus Deutschland kommend veröffentlicht werden. Anhand dieser Daten und der Folgepublikationen lassen sich viele Therapiealgorithmen auf ihre Validität im „real life“ überprüfen, zudem ermöglicht der große Pool an Daten die Überprüfung weiterer wichtiger klinischer Fragestellungen.

Aktuell werden zudem Gewebsblöcke gesammelt, um erstmalig auch im größeren Umfang translationale Fragestellungen bei dieser Entität zu untersuchen. Möglicherweise wird das Studiendesign (retrospektives multizentrisches Vorgehen mit mehr als 30 Zentren über einen größeren Zeitraum) als Blaupause dienen, um auch für andere seltene Erkran- kungen (z. B. Sarkome) in Zukunft erstmals eine solide Datenlage zu generieren.

\begin{tabular}{l}
\hline Erstmalig werden bei der \\
Entität Vulvakarzinom im \\
größeren Umfang translationale \\
Fragen untersucht
\end{tabular}

Kontrovers diskutiert wird zurzeit beim Vulvakarzinom insbesondere der erforderliche Resektionsrand. Ähnlich wie beim Mammakarzinom kann hier in den letzten Jahren beobachtet werden, dass die in Leitlinien empfohlenen Sicherheitsabstände stetig abnehmend sind. So wird in der aktuellen Leitlinie ein Sicherheitsabstand von mindestens $3 \mathrm{~mm}$ gefordert, allerdings ist diese Empfehlung nicht evidenzbasiert, sondern ein klinischer Kompromiss. Trotzdem ist dieser noch weit entfernt von der Noink-on-tumor-Strategie bei der Mamma.

Sollte eine Operation aus Komorbiditätsgründen oder wegen der Tumorausdehnung nicht möglich sein, besteht mit der hochwirksamen Strahlentherapie ein valide Alternative, über die Ihnen die Ulmer Kollegen Wiegel und Bottke Auskunft geben werden.

Besonders besorgniserregend ist die Zunahme der Erkrankungshäufigkeit bei den jüngeren Frauen. Für sie spielt der sexuelle Funktionserhalt trotz therapeutischer Maßnahmen eine wesentliche Rolle. In den letzten Jahren wurden verschiedene Möglichkeiten der plastischen Rekonstruktion nach Exzision publiziert, einen guten Überblick hierzu gibt Ihnen der Artikel der Münchner Kollegen. Wie viel Radikalität im Bereich des Lymphknotenstaging - mit all seinen belasten- den Folgen - noch erforderlich ist, wird von Florian Ebner et al. erörtert.

Aufgrund der stark mit Scham belasteten Lokalisation stellen sich Patientinnen im Senium sehr häufig mit malignen Erkrankungen im weit fortgeschrittenen Stadium vor. Auch hier ist es an uns Frauenärzten, die Patientin zur regelmäßigen Vorsorge auch nach der Überschreitung des 70. Lebensjahres zu ermutigen.

Da sich die typischen Symptome der malignen Erkrankung nicht von denen der benignen Differenzialdiagnosen unterscheiden, ist ein guter klinischer Blick und das Wissen um die Differenzialdiagnosen entscheidend. Hier kommt den niedergelassenen Kollegen eine wesentliche Funktion zu, Unterstützung bietet dafür ein reich bebilderter Artikel der Kollegen der Uniklinik in Düsseldorf.

Trotz einer modernen Therapie erleiden bis $\mathrm{zu} 50 \%$ der Patientinnen langfristig ein Rezidiv. Welche therapeutischen Optionen in dieser Situation evidenzbasiert sind, wird einem Artikel von Linn Wölber aus Hamburg verdeutlicht werden.

Mit besten Gruß aus Ulm

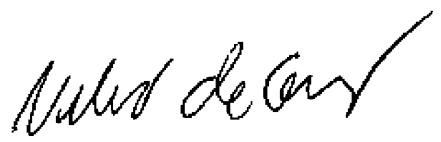

Dr. Nikolaus de Gregorio

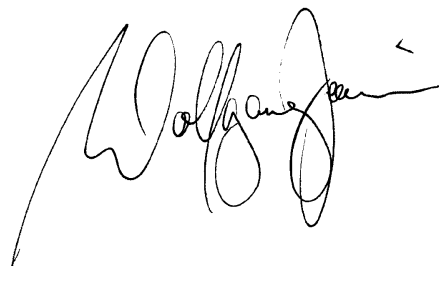

Prof. Dr. Wolfgang Janni 


\section{Korrespondenzadresse}

\begin{tabular}{|c|c|}
\hline & $\begin{array}{l}\text { Dr. N. de Gregorio } \\
\text { Universitätsfrauenklinik Ulm } \\
\text { Prittwitzstr.43,89075 Ulm, } \\
\text { Deutschland } \\
\text { Nikolaus.de-gregorio@ } \\
\text { uniklinik-ulm.de }\end{array}$ \\
\hline & $\begin{array}{l}\text { Prof. Dr. W. Janni } \\
\text { Universitätsfrauenklinik UIm } \\
\text { Prittwitzstr. 43,89075 Ulm, } \\
\text { Deutschland } \\
\text { Wolfgang.Janni@ } \\
\text { uniklinik-ulm.de }\end{array}$ \\
\hline
\end{tabular}

Interessenkonflikt. N. de Gregorio und W. Janni geben an, dass kein Interessenkonflikt besteht.

\section{Literatur}

1. Mahner S, Jueckstock J, Hilpert F et al. (2015) Adjuvant therapy in lymph node-positive vulvar cancer: The AGO-CaRE-1 study. J Natl Cancer Inst 107. doi:10.1093/jnci/dju426 